\title{
An IQC-Based Stability Criterion for Systems with Slowly Varying Parameters
}

\author{
Anders Helmerssonn \\ Department of Electrical Engineering \\ Linköping University, S-581 83 Linköping, Sweden \\ www: http://www. control.isy.liu.se \\ email: andersh@isy.liu.se
}

LiTH-ISY-R-1979

September 10, 1997

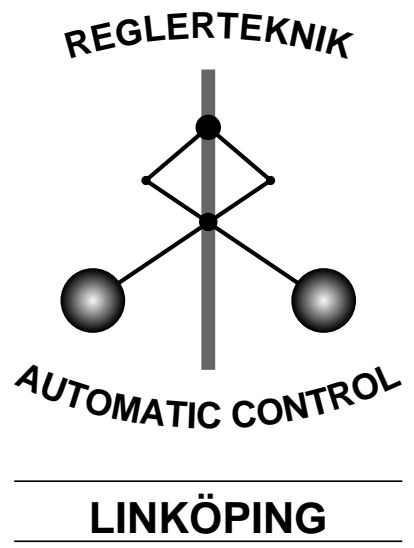

Technical reports from the Automatic Control group in Linköping are available as UNIX-compressed Postscript files by anonymous ftp at the address 130.236.20.24 (ftp.control.isy.liu.se). 


\title{
An IQC-Based Stability Criterion for Systems with Slowly Varying Parameters*
}

\author{
Anders Helmersson \\ Department of Electrical Engineering \\ Linköping University, S-581 83 Linköping, Sweden \\ www: http://www.control.isy.liu.se \\ email: andersh@isy.liu.se
}

Submitted to ACC 1998

September 10, 1997

\begin{abstract}
An integral quadratic constraints (IQC) is introduced for stability analysis of linear systems with slowly varying parameters. The parameters are assumed to be bounded and with bounded derivatives. Other types of uncertainties can be included in the problem. The new criterion yields significantly less conservative bounds than previously proposed criteria.
\end{abstract}

Keywords: Stability analysis, robustness, linear time-varying, integral quadratic constraints.

\section{Introduction}

Integral Quadratic Constraints (IQC) has recently been proposed by Megretski and Rantzer as a unified approach to robustness analysis [5].

We will here elaborate on IQCs for verifying stability of linear systems with slowly varying parameters. We assume that the parameters are bounded as well as are their derivatives. An IQC-based stability criterion for slowly-varying parameters (uncertainties) has been proposed by Jönsson and Rantzer [3, 2]. The IQC proposed in this paper exploits more of the structure of the problem than the previous criterion. Since higher flexibility in the IQC multiplier can be allowed the new criterion gives less conservative bounds. This is illustrated by two examples.

The paper is organized as follows. Integral Quadratic Constraints are briefly introduced in section 2. In section 3 , the main result is elaborated including the swapping lemma and choice of multipliers. The new algorithm is applied to two examples in section 4 . The conclusions are given in section 5 .

\footnotetext{
* This work was supported by the Swedish National Board for Industrial and Technical Development (NUTEK), which is gratefully acknowledged
} 


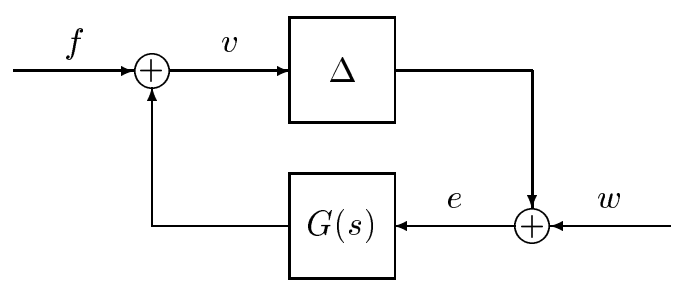

Figure 1: Basic feedback configuration.

\section{$1.1 \quad$ Notation}

For matrices $A^{*}$ denotes the complex conjugate transpose.

$\mathcal{R} \mathcal{H}_{\infty}$ denotes denotes stable real-rational transfer functions; $\mathcal{L}_{2}$ denotes the Hilbert space of measurable functions $\mathbb{R} \rightarrow \mathbb{R}^{n}$ satisfying

$$
\|f\|_{2}^{2}=\int_{-\infty}^{\infty}|f(t)|_{2}^{2} d t<\infty
$$

This is a subspace of $\mathcal{L}_{2 e}$, whose members only need to be square integrable on finite intervals.

\section{Integral Quadratic Constraints}

The integral quadratic constraints (IQCs) has been proposed for robustness analysis [5]. The IQC states a stability criterion for the interconnection of a stable system $M \in \mathcal{R} \mathcal{H}_{\infty}$ and a bounded causal operator $\Delta$, see figure 1 .

$$
\left\{\begin{array}{l}
v=M w+f \\
w=\Delta v+e
\end{array}\right.
$$

We say that the interconnection of $M$ and $\Delta$ is well-posed if the map $(v, w) \rightarrow$ $(e, f)$ defined by $(1)$ has a causal inverse on $\mathcal{L}_{2 e}$. The interconnection is stable if, in addition, the inverse is bounded, that is if there exists a constant $C$ such that

$$
\int_{0}^{T}\left(|v(t)|^{2}+|w(t)|^{2}\right) d t \leq C \int_{0}^{T}\left(|f(t)|^{2}+|e(t)|^{2}\right) d t
$$

for any $T \geq 0$ and for any solution of (1).

Depending on the particular application, various versions of IQCs are available. Two signals $w \in \mathcal{L}_{2}[0, \infty)$ and $v \in \mathcal{L}_{2}[0, \infty)$ are said to satisfy the IQC defined by $\Pi$, if

$$
\int_{\infty}^{\infty}\left[\begin{array}{c}
\hat{v}(j \omega) \\
\hat{w}(j \omega)
\end{array}\right]^{*} \Pi(j \omega)\left[\begin{array}{c}
\hat{v}(j \omega) \\
\hat{w}(j \omega)
\end{array}\right] d \omega \geq 0
$$

where absolute integrability is assumed. Here $\hat{v}(j \omega)$ and $\hat{v}(j \omega)$ represent the harmonic spectrum of the signals $v$ and $w$ at the frequency $\omega$. In principle $\Pi: j \mathbb{R} \rightarrow \mathbb{C}$ can be any measurable Hermitian-valued function. In most applications, however, it is sufficient to use rational functions that are bounded on the imaginary axis. 
A time-domain form of (2) is

$$
\int_{0}^{\infty} \sigma\left(x_{\pi}(t), v(t), w(t)\right) d t \geq 0
$$

where $\sigma$ is a quadratic form, and $x_{\pi}$ is defined by

$$
\dot{x}_{\pi}(t)=A_{\pi} x_{\pi}(t)+B_{v} v(t)+B_{w} w(t), \quad x_{\pi}(0)=0
$$

where $A_{\pi}$ is a Hurwitz matrix.

The main theorem from [5] goes as follows

Theorem $1([5])$ Let $G \in \mathcal{R H}_{\infty}$ and let $\Delta$ be a bounded causal operator. Assume that:

i) for every $\tau \in[0,1]$, the interconnection of $G$ and $\tau \Delta$ is well-posed;

ii) for every $\tau \in[0,1]$, the IQC defined by $\Pi$ is satisfied by $\tau \Delta$;

iii) there exists $\epsilon>0$ such that

$$
\left[\begin{array}{c}
G(j \omega) \\
I
\end{array}\right]^{*} \Pi(j \omega)\left[\begin{array}{c}
G(j \omega) \\
I
\end{array}\right] \leq-\epsilon I, \quad \forall \omega \in \mathbb{R}
$$

Then the feedback interconnection of $G$ and $\Delta$ is stable.

Note that if the upper left corner, $\Pi_{11}$, of $\Pi$ is positive semidefinite then $\Delta=0$ satisfies (2). If further the lower right corner, $\Pi_{22}$, is negative semidefinite then any convex combination of $\Delta$ 's satisfying (2) also satisfies the IQC. Thus, $\Pi_{11} \geq 0$ and $\Pi_{22} \leq 0$ imply that $\tau \Delta$ satisfies (2) for $\tau \in[0,1]$ if and only if $\Delta$ does so. This simplifies assumption ii).

This follows by the fact that

$$
\begin{aligned}
& {\left[\begin{array}{c}
I \\
\lambda \Delta_{1}+(1-\lambda) \Delta_{2}
\end{array}\right]\left[\begin{array}{ll}
\Pi_{11} & \Pi_{12} \\
\Pi_{12}^{*} & \Pi_{22}
\end{array}\right]\left[\begin{array}{c}
I \\
\lambda \Delta_{1}+(1-\lambda) \Delta_{2}
\end{array}\right]} \\
& =\lambda\left[\begin{array}{c}
I \\
\Delta_{1}
\end{array}\right]\left[\begin{array}{ll}
\Pi_{11} & \Pi_{12} \\
\Pi_{12}^{*} & \Pi_{22}
\end{array}\right]\left[\begin{array}{c}
I \\
\Delta_{1}
\end{array}\right]+(1-\lambda)\left[\begin{array}{c}
I \\
\Delta_{2}
\end{array}\right]\left[\begin{array}{cc}
\Pi_{11} & \Pi_{12} \\
\Pi_{12}^{*} & \Pi_{22}
\end{array}\right]\left[\begin{array}{c}
I \\
\Delta_{2}
\end{array}\right] \\
& -\lambda(1-\lambda)\left[\begin{array}{c}
0 \\
\Delta_{1}-\Delta_{2}
\end{array}\right]\left[\begin{array}{cc}
0 & 0 \\
0 & \Pi_{22}
\end{array}\right]\left[\begin{array}{c}
0 \\
\Delta_{1}-\Delta_{2}
\end{array}\right] \\
& \geq \lambda\left[\begin{array}{c}
I \\
\Delta_{1}
\end{array}\right]\left[\begin{array}{ll}
\Pi_{11} & \Pi_{12} \\
\Pi_{12}^{*} & \Pi_{22}
\end{array}\right]\left[\begin{array}{c}
I \\
\Delta_{1}
\end{array}\right]+(1-\lambda)\left[\begin{array}{c}
I \\
\Delta_{2}
\end{array}\right]\left[\begin{array}{ll}
\Pi_{11} & \Pi_{12} \\
\Pi_{12}^{*} & \Pi_{22}
\end{array}\right]\left[\begin{array}{c}
I \\
\Delta_{2}
\end{array}\right]
\end{aligned}
$$

if $\lambda \in[0,1]$ and $\Pi_{22} \leq 0$. Also, $\Pi_{11} \geq 0$ assures that the inequality is satisfied for $\Delta=0$.

The search for multipliers, $\Pi$, can be carried out as a convex optimization problem by parametrizing

$$
\Pi(j \omega)=\sum_{i} x_{i} \Pi_{i}(j \omega)
$$

where $x_{i}$ are positive real parameters and $\Pi_{i}$ is a set of basis multipliers. By applying the Kalman-Yakubovich-Popov lemma $[7,8,6]$, the search for $x_{i}$, can be implemented using linear matrix inequalities (LMIs). 


\section{$3 \quad$ Slowly Varying Uncertainties}

We will here propose an IQC for verifying stability of linear system with slowly varying uncertainties. We define slowly varying uncertainties such that their rate of change $\dot{\Delta}$ is norm-bounded by $d$, that is $\|\dot{\Delta}\| \leq d$.

The main tool for this analysis is the swapping lemma that tells how a linear operator on state-space form commutes with a time-varying uncertainty.

\subsection{Swapping Lemma}

The following swapping lemma is essentially from [2].

Lemma 1 (Swapping Lemma) Suppose that $\hat{\Delta}$ has the derivative $\dot{\hat{\Delta}}$ and $u \in$ $\mathcal{L}_{2}$. Further let $T \in \mathcal{R H}_{\infty}$ :

$$
\begin{aligned}
T(s) & =\hat{D}+\hat{C}(s I-\hat{A})^{-1} \hat{B} \\
U(s) & =\hat{C}(s I-\hat{A})^{-1} \\
V(s) & =(s I-\hat{A})^{-1} \hat{B}
\end{aligned}
$$

such that

$$
\left[\begin{array}{ll}
\hat{A} & \hat{B} \\
\hat{C} & \hat{D}
\end{array}\right]\left[\begin{array}{cc}
\hat{\Delta} & 0 \\
0 & \Delta
\end{array}\right]=\left[\begin{array}{cc}
\hat{\Delta} & 0 \\
0 & \bar{\Delta}
\end{array}\right]\left[\begin{array}{ll}
\hat{A} & \hat{B} \\
\hat{C} & \hat{D}
\end{array}\right]
$$

Then

$$
\bar{\Delta} T(s)=(T(s) \Delta u+U(s) \dot{\hat{\Delta}} V(s)) u .
$$

Proof: $\quad$ Let $s=\frac{d}{d t}$. We note that

$$
(s I-\hat{A}) \hat{\Delta} V(s) u=\dot{\hat{\Delta}} V(s) u+\hat{\Delta} \bar{B} u=\dot{\hat{\Delta}} V(s) u+\bar{B} \Delta u .
$$

Let $U(s)$ operate from the left on (7). After addition of $\bar{\Delta} \hat{D} u=\hat{D} \Delta u$, we get

$$
(\bar{\Delta} \hat{D}+\hat{C} \hat{\Delta} V(s)) u=(U(s) \dot{\hat{\Delta}} V(s)+\hat{D} \Delta+U(s) \bar{B} \Delta) u,
$$

which is equivalent to (6).

Note that we can generalize the swapping lemma to also apply to a more general set of linear operators $\hat{\Delta}$ for which we define $\dot{\hat{\Delta}}$ by

$$
\frac{d}{d t}(\hat{\Delta} v)=\dot{\hat{\Delta}} v+\hat{\Delta} \dot{v}
$$

for all $v, \dot{v} \in \mathcal{L}_{2}$.

Also note that the block-diagonal structure of $T, V$ and $U$ is implicitly defined by the commuting equation (5).

The set of uncertainties, $\boldsymbol{\Delta}$, can be assumed to have a blockdiagonal structure, possibly with repeated sub-blocks:

$$
\boldsymbol{\Delta}=\left\{\Delta=\operatorname{diag}\left[I_{n_{1}} \otimes \Delta_{1}, \ldots, I_{n_{f}} \otimes \Delta_{f}\right]\right\}
$$


where $\otimes$ denotes the Kronecker product. The uncertainties consist of $f$ diagonal blocks, which could be either dynamic (complex) or parametric (real). For parametric blocks also $\Delta_{i}=\Delta_{i}^{*}$ applies. It is easy to show that for any $\Delta_{i} \in$ $\mathbb{C}^{k_{i} \times k_{i}}$ and $D_{i} \in \mathbb{C}^{n_{i} \times n_{i}}$ the following commutative equation holds:

$$
\left(I_{n_{i}} \otimes \Delta_{i}\right)\left(D_{i} \otimes I_{k_{i}}\right)=\left(D_{i} \otimes I_{k_{i}}\right)\left(I_{n_{i}} \otimes \Delta_{i}\right)
$$

In the paper the structure of $\boldsymbol{\Delta}$ is implicit, and instead the structure is defined by commuting properties, such as (5) and (8).

For instance, if we assume that $\Delta=\operatorname{diag}\left[\delta_{1} I_{n_{1}}, \Delta_{2}\right]$ where $\delta_{1}$ is a slowly varying parameter and $\Delta_{2}$ contains remaining uncertainties, a natural choice of $T$ would be to use

$$
\left[\begin{array}{ll}
\hat{A} & \hat{B} \\
\hat{C} & \hat{D}
\end{array}\right]=\left[\begin{array}{c|cc}
\hat{A} & \hat{B} & 0 \\
\hline I & 0 & 0 \\
0 & I & 0 \\
0 & 0 & I
\end{array}\right]
$$

Then $\hat{\Delta}=\delta_{1} I_{k_{1}}, \bar{\Delta}=\operatorname{diag}\left[\delta_{1} I_{k_{1}}, \Delta\right]=\operatorname{diag}\left[\delta_{1} I_{n_{1}+k_{1}}, \Delta_{2}\right]$, where $k_{1}$ is the dimension of $\hat{A}$. In general $\hat{\Delta}$ contains the slowly varying parameters in $\Delta$ possibly (differently) repeated.

\subsection{IQC Formulation}

We will study a stability criterion for the system $x=\Delta G x$ where $\Delta$ and $\dot{\hat{\Delta}}$ are norm-bounded. Consider the following IQC-like matrix inequality.

$$
\left[\begin{array}{cc}
T(j \omega) G(j \omega) & 0 \\
V(j \omega) G(j \omega) & 0 \\
\hline T(j \omega) & U(j \omega) \\
0 & I
\end{array}\right]^{*} \tilde{\Pi}\left[\begin{array}{cc}
T(j \omega) G(j \omega) & 0 \\
\frac{V(j \omega) G(j \omega)}{2(j \omega)} & 0 \\
\hline 0 & I j \omega)
\end{array}\right]<0
$$

where $\tilde{\Pi}=\tilde{\Pi}^{*}$ is a static matrix. We can rewrite (10) as a proper IQC for the augmented system $\left[\begin{array}{ll}G & 0\end{array}\right]$ :

$$
\left[\begin{array}{rr}
G(j \omega) & 0 \\
\hline I & 0 \\
0 & I
\end{array}\right]^{*} \Pi(j \omega)\left[\begin{array}{cc}
G(j \omega) & 0 \\
\hline I & 0 \\
0 & I
\end{array}\right]<0, \quad \omega \in \mathbb{R} \cup\{\infty\}
$$

where

$$
\Pi=\left[\begin{array}{ll}
\Pi_{11} & \Pi_{12} \\
\Pi_{12}^{*} & \Pi_{22}
\end{array}\right]=\left[\begin{array}{ccc}
T & 0 & 0 \\
V & 0 & 0 \\
\hline 0 & T & U \\
0 & 0 & I
\end{array}\right]^{*} \tilde{\Pi}\left[\begin{array}{ccc}
T & 0 & 0 \\
V & 0 & 0 \\
\hline 0 & T & U \\
0 & 0 & I
\end{array}\right] .
$$

The multiplier defined by (12) satisfies the IQC for the augmented uncertainty given by $\left[\begin{array}{c}\Delta \\ \dot{\hat{\Delta}} V\end{array}\right]$. To show this we first observe that the augmented uncertainty 
is bounded since $T \in \mathcal{R} \mathcal{H}_{\infty}$ and, consequently, $\dot{\hat{\Delta}} V$ is bounded if $\dot{\hat{\Delta}}$ is such. Using the swapping lemma, we obtain

$$
w=\left[\begin{array}{ccc}
T & 0 & 0 \\
V & 0 & 0 \\
0 & T & U \\
0 & 0 & I
\end{array}\right]\left[\begin{array}{c}
I \\
\Delta \\
\dot{\hat{\Delta}} V
\end{array}\right] v=\left[\begin{array}{c}
T \\
V \\
T \Delta+U \dot{\hat{\Delta}} V \\
\dot{\hat{\Delta}} V
\end{array}\right] v=\left[\begin{array}{c}
T \\
V \\
\bar{\Delta} T \\
\dot{\hat{\Delta}} V
\end{array}\right] v .
$$

Thus (3) becomes

$$
\begin{aligned}
& \int_{0}^{\infty} w(t)^{*} \tilde{\Pi} w(t) d t \\
& =\int_{0}^{\infty}\left[\begin{array}{l}
w_{T}(t) \\
w_{V}(t)
\end{array}\right]^{*}\left[\begin{array}{cc}
I & 0 \\
0 & I \\
\hline \bar{\Delta}(t) & 0 \\
0 & \dot{\hat{\Delta}}(t)
\end{array}\right]^{*} \tilde{\Pi}\left[\begin{array}{cc}
I & 0 \\
0 & I \\
\hline \bar{\Delta}(t) & 0 \\
0 & \dot{\hat{\Delta}}(t)
\end{array}\right]\left[\begin{array}{c}
w_{T}(t) \\
w_{V}(t)
\end{array}\right] d t \geq 0
\end{aligned}
$$

where $w_{T}=T v$ and $w_{V}=V v$ are defined by

$$
\begin{aligned}
\dot{\hat{x}}(t) & =\hat{A} \hat{x}(t)+\hat{B} v(t), \quad \hat{x}(0)=0 \\
{\left[\begin{array}{l}
w_{T}(t) \\
w_{V}(t)
\end{array}\right] } & =\left[\begin{array}{cc}
\hat{C} & \hat{D} \\
I & 0
\end{array}\right]\left[\begin{array}{l}
\hat{x}(t) \\
v(t)
\end{array}\right] .
\end{aligned}
$$

Using (5) we have

$$
\left[\begin{array}{cc}
\bar{\Delta}(t) & 0 \\
0 & \dot{\hat{\Delta}}(t)
\end{array}\right]\left[\begin{array}{cc}
\hat{C} & \hat{D} \\
I & 0
\end{array}\right]=\left[\begin{array}{ccc}
\hat{C} & \hat{D} & 0 \\
0 & 0 & I
\end{array}\right]\left[\begin{array}{cc}
\hat{\Delta}(t) & 0 \\
0 & \Delta(t) \\
\hat{\hat{\Delta}}(t) & 0
\end{array}\right]
$$

Consequently, (13) is equivalent to

$$
\int_{0}^{\infty}\left[\begin{array}{l}
\hat{x}(t) \\
v(t)
\end{array}\right]^{*}\left[\begin{array}{cc}
I & 0 \\
0 & I \\
\hline \hat{\Delta}(t) & 0 \\
0 & \Delta(t) \\
\dot{\hat{\Delta}}(t) & 0
\end{array}\right]^{*}\left[\begin{array}{cc}
I & 0 \\
0 & I \\
\hline \hat{\Pi}(t) & 0 \\
0 & \Delta(t) \\
\dot{\hat{\Delta}}(t) & 0
\end{array}\right]\left[\begin{array}{c}
\hat{x}(t) \\
v(t)
\end{array}\right] d t \geq 0
$$

for any $v \in \mathcal{L}_{2}$ and $\hat{x}$ as defined by (14) and where

$$
\tilde{\tilde{\Pi}}=\left[\begin{array}{ll}
\tilde{\tilde{\Pi}}_{11} & \tilde{\tilde{\Pi}}_{12} \\
\tilde{\tilde{\Pi}}_{12}^{*} & \tilde{\tilde{\Pi}}_{22}
\end{array}\right]=\left[\begin{array}{cc|ccc}
\hat{C} & \hat{D} & 0 & 0 & 0 \\
I & 0 & 0 & 0 & 0 \\
\hline 0 & 0 & \hat{C} & \hat{D} & 0 \\
0 & 0 & 0 & 0 & I
\end{array}\right]^{*}\left[\begin{array}{ll}
\tilde{\Pi}_{11} & \tilde{\Pi}_{12} \\
\tilde{\Pi}_{12}^{*} & \tilde{\Pi}_{22}
\end{array}\right]\left[\begin{array}{cc|ccc}
\hat{C} & \hat{D} & 0 & 0 & 0 \\
I & 0 & 0 & 0 & 0 \\
\hline 0 & 0 & \hat{C} & \hat{D} & 0 \\
0 & 0 & 0 & 0 & I
\end{array}\right] .
$$

If $\tilde{\tilde{\Pi}}_{11} \geq 0$ and $\tilde{\tilde{\Pi}}_{22} \leq 0$, then the inequality (15) is satisfied for any time-varying convex combinations of $\hat{\Delta}$ 's that satisfy the same inequality. See the remark on theorem 1. 
We will now show that there exists such a multiplier, $\tilde{\tilde{\Pi}}$, if $\Pi_{11}(j \omega) \geq 0$ and $\Pi_{22}(j \omega) \leq 0$ for all $\omega \in \mathbb{R}$. We assume that $\hat{A}$ is Hurwitz and that the state-space representation of $T$ is minimal so that $(\hat{A}, \hat{B})$ is controllable.

The positive semidefiniteness of $\Pi_{11}(j \omega)$ is equivalent to

$$
\left[\begin{array}{l}
T(j \omega) \\
V(j \omega)
\end{array}\right]^{*} \tilde{\Pi}_{11}\left[\begin{array}{l}
T(j \omega) \\
V(j \omega)
\end{array}\right]=\left[\begin{array}{c}
(j \omega I-\hat{A})^{-1} \hat{B} \\
I
\end{array}\right]^{*} \tilde{\tilde{\Pi}}_{11}\left[\begin{array}{c}
(j \omega I-\hat{A})^{-1} \hat{B} \\
I
\end{array}\right] \geq 0
$$

for all $\omega \in \mathbb{R}$. Using the Kalman-Yakubovich-Popov lemma $[7,8,6]$, this inequality holds if and only if there exists $Y=Y^{*}$ such that

$$
\tilde{\tilde{\Pi}}_{11}+\left[\begin{array}{cc}
Y \hat{A}+\hat{A}^{*} Y & Y \hat{B} \\
\hat{B}^{*} Y & 0
\end{array}\right] \geq 0
$$

Thus, if $\Pi_{11}(j \omega) \geq 0$ for all $\omega \in \mathbb{R}$ we can modify $\tilde{\tilde{\Pi}}_{11}$ to become positive semidefinite by adding

$$
\tilde{\tilde{\Pi}}_{11 Y}=\left[\begin{array}{cc}
Y \hat{A}+\hat{A}^{*} Y & Y \hat{B} \\
\hat{B}^{*} Y & 0
\end{array}\right] .
$$

This does not affect neither (11) nor (15), since

$$
\left[\begin{array}{c}
(j \omega I-\hat{A})^{-1} \hat{B} \\
I
\end{array}\right]^{*} \tilde{\tilde{\Pi}}_{11 Y}\left[\begin{array}{c}
(j \omega I-\hat{A})^{-1} \hat{B} \\
I
\end{array}\right]=0 .
$$

Using the same sequence of arguments

$$
\begin{gathered}
\Pi_{22}(j \omega)=\left[\begin{array}{cc}
T(j \omega) & U(j \omega) \\
0 & I
\end{array}\right]^{*} \tilde{\Pi}_{22}\left[\begin{array}{cc}
T(j \omega) & U(j \omega) \\
0 & I
\end{array}\right] \\
\quad=\left[\begin{array}{cc}
(j \omega-\hat{A})^{-1} \hat{B} & (j \omega-\hat{A})^{-1} \\
I & 0 \\
0 & I
\end{array}\right]^{*} \tilde{\tilde{\Pi}}_{22}\left[\begin{array}{cc}
(j \omega-\hat{A})^{-1} \hat{B} & (j \omega-\hat{A})^{-1} \\
I & 0 \\
0 & I
\end{array}\right] \leq 0
\end{gathered}
$$

holds for all $\omega \in \mathbb{R}$, if and only if there exists $X=X^{*}$ such that

$$
\tilde{\tilde{\Pi}}_{22}+\left[\begin{array}{ccc}
X \hat{A}+\hat{A}^{*} X & X \hat{B} & X \\
\hat{B}^{*} X & 0 & 0 \\
X & 0 & 0
\end{array}\right] \leq 0 .
$$

Thus we can modify $\tilde{\tilde{\Pi}}_{22}$ to become negative semidefinite if and only if $\Pi_{22}(j \omega) \leq$ 0 for all $\omega \in \mathbb{R}$. Further, we can show that also (15) is unaffected by the same modification of $\tilde{\tilde{\Pi}}_{22}$, since

$$
\begin{aligned}
& \int_{0}^{\infty}\left[\begin{array}{l}
\hat{x}(t) \\
v(t)
\end{array}\right]^{*}\left[\begin{array}{cc}
\hat{\Delta}(t) & 0 \\
0 & \Delta(t) \\
\dot{\hat{\Delta}}(t) & 0
\end{array}\right]^{*}\left[\begin{array}{ccc}
X \hat{A}+\hat{A}^{*} X & X \hat{B} & X \\
\hat{B}^{*} X & 0 & 0 \\
X & 0 & 0
\end{array}\right]\left[\begin{array}{cc}
\hat{\Delta}(t) & 0 \\
0 & \Delta(t) \\
\dot{\hat{\Delta}}(t) & 0
\end{array}\right]\left[\begin{array}{c}
\hat{x}(t) \\
v(t)
\end{array}\right] d t \\
& =\int_{0}^{\infty} \hat{x}(t)^{*} \hat{\Delta}(t)^{*} X(\hat{A} \hat{\Delta}(t) \hat{x}(t)+\hat{B} \Delta(t) v(t)+\dot{\hat{\Delta}}(t) \hat{x}(t))+()^{*} d t \\
& =\int_{0}^{\infty} \hat{x}(t)^{*} \hat{\Delta}(t)^{*} X(\hat{\Delta}(t) \hat{A} \hat{x}(t)+\hat{\Delta} \hat{B} v(t)+\dot{\hat{\Delta}}(t) \hat{x}(t))+()^{*} d t \\
& =\int_{0}^{\infty} \frac{d}{d t}\left(\hat{x}(t)^{*} \hat{\Delta}(t)^{*} X \hat{\Delta}(t) \hat{x}(t)\right) d t \\
& =\lim _{t \rightarrow \infty} \hat{x}(t)^{*} \hat{\Delta}(t)^{*} X \hat{\Delta}(t) \hat{x}(t)-\hat{x}(0)^{*} \hat{\Delta}(0)^{*} X \hat{\Delta}(0) \hat{x}(0)=0
\end{aligned}
$$


since $\hat{x}, \dot{\hat{x}} \in \mathcal{L}_{2}$ and $\hat{x}(0)=0$. Here we have used ()$^{*}$ to denote the transpose of the previous terms.

We have shown that if $\Pi_{11}(j \omega) \geq 0$ and $\Pi_{22}(j \omega) \leq 0$ for $\omega \in \mathbb{R}$, then we can find an equivalent IQC with $\tilde{\tilde{\Pi}}$ such that $\tilde{\tilde{\Pi}}_{11} \geq 0$ and $\tilde{\tilde{\Pi}}_{22} \leq 0$. Thus (13) and (15) both hold for any time-varying convex-cone combination such that

$$
\begin{aligned}
& \hat{\Delta}(t)=\sum_{i} \lambda_{i}(t) \hat{\Delta}_{i}(t) \\
& \dot{\hat{\Delta}}(t)=\sum_{i} \lambda_{i}(t) \dot{\hat{\Delta}}_{i}(t)
\end{aligned}
$$

for $\lambda_{i}(t) \in[0,1]$ and $\sum_{i} \lambda_{i}(t) \leq 1$. Note that (19) constrains $\lambda_{i}$ dynamically since $\hat{\Delta}$ and $\dot{\hat{\Delta}}$ are related. Specifically, it follows that $\sum_{i} \dot{\lambda}_{i}(t) \hat{\Delta}_{i}(t)=0$. For instance, if $\dot{\hat{\Delta}}_{i}=0$ then only time-invariant $\hat{\Delta}$ are allowed.

If we have a multiplier $\tilde{\tilde{\Pi}}$ such that

$$
\left[\begin{array}{cc}
I & 0 \\
0 & I \\
\hline \bar{\Delta}_{i}(t) & 0 \\
0 & \hat{\Delta}_{i}(t)
\end{array}\right]^{*} \tilde{\Pi}\left[\begin{array}{cc}
I & 0 \\
0 & I \\
\hline \bar{\Delta}_{i}(t) & 0 \\
0 & \hat{\Delta}_{i}(t)
\end{array}\right] \geq 0, \quad \forall i, t
$$

that satisfies (11) and if $\Pi_{11}(j \omega) \geq 0$ and $\Pi_{22}(j \omega) \leq 0$ for all $\omega \in \mathbb{R}$, then the system is verified to be stable for any time-varying convex-cone combination of the $\bar{\Delta}_{i}$ and $\dot{\hat{\Delta}}_{i}$.

For instance, if $\bar{\Delta}=\delta I$ and $\dot{\hat{\Delta}}=\dot{\delta} I$ such that $|\delta| \leq 1$ and $|\dot{\delta}|<d$, then we may use

$$
\tilde{\Pi}=\left[\begin{array}{cc|cc}
P_{1} & 0 & \Gamma_{1} & 0 \\
0 & d^{2} P_{2} & 0 & \Gamma_{2} \\
\hline \Gamma_{1}^{*} & 0 & -P_{1}-\varepsilon_{1} I & 0 \\
0 & \Gamma_{2}^{*} & 0 & -P_{2}
\end{array}\right]
$$

where $P_{1}=P_{1}^{*}, P_{2}=P_{2}^{*} \geq 0, \Gamma_{1}=-\Gamma_{1}^{*}$ and $\Gamma_{2}=-\Gamma_{2}^{*}$. We do not require $P_{1}$ to be positive semidefinite, while $P_{2}$ must be so in order not to violate $\Pi_{11}(j \omega) \geq 0$ and $\Pi_{22}(j \omega) \leq 0$. Since, (21) satisfies (20) for $\delta= \pm\left[1-\varepsilon_{2}, 1+\varepsilon_{2}\right]$ and $|\dot{\delta}| \leq d$ we can allow any time-varying convex-cone combination if the IQC holds. The variable $\varepsilon_{1}$ is an arbitrarily small positive constant that is chosen in order to allow a variation of $\delta$ within small bands of width $2 \varepsilon_{2}$ around \pm 1 .

To summarize, we have arrived at the following theorem:

Theorem 2 Assume that $\bar{\Delta}_{i}$ and $\dot{\hat{\Delta}}_{i}$ satisfy (13) and that the interconnection of $\Delta$ and $G(s)$ is well-posed for every $\Delta$ in the convex cone defined by $\Delta_{i}$. Then the system $u=\Delta G(s) u$ is asymptotically stable for any time-varying convexcone combination of $\bar{\Delta}_{i}$ and $\dot{\hat{\Delta}}_{i}$ if there exists a $\Pi$ as defined by (12) such that

$$
\begin{aligned}
& {\left[\begin{array}{cc}
G(j \omega) & 0 \\
\hline I & 0 \\
0 & I
\end{array}\right] \Pi(j \omega)\left[\begin{array}{cc}
G(j \omega) & 0 \\
I & 0 \\
0 & I
\end{array}\right]<0} \\
& \Pi_{11}(j \omega) \geq 0 \\
& \Pi_{22}(j \omega) \leq 0
\end{aligned}
$$

hold for all $\omega \in \mathbb{R} \cup\{\infty\}$. 


\subsection{Comparison with Previous Criterion}

Jönsson and Rantzer proposed an IQC for the stability analysis of linear system with slowly varying parameters $[3,2]$ :

$$
\left[\begin{array}{c}
M \\
I
\end{array}\right]^{*}\left[\begin{array}{cc}
R^{*} R+V_{R}^{*} V_{R}+d^{2} V_{S}^{*} V_{S}+U_{S} U_{S}^{*} & S-S^{*} \\
S^{*}-S & -R^{*}\left(I-U_{R} U_{R}^{*}\right) R
\end{array}\right]\left[\begin{array}{c}
M \\
I
\end{array}\right]<0,
$$

where

$$
\begin{array}{rlrl}
R & =D_{R}+C_{R}\left(s I-A_{R}\right)^{-1} B_{R} & S & =D_{S}+C_{S}\left(s I-A_{S}\right)^{-1} B_{S} \\
U_{R} & =C_{R}\left(s I-A_{R}\right)^{-1} & U_{S} & =C_{S}\left(s I-A_{S}\right)^{-1} \\
V_{R} & =\left(s I-A_{R}\right)^{-1} B_{R} & V_{S} & =\left(s I-A_{S}\right)^{-1} B_{S}
\end{array}
$$

such that $R$ and $S$ are both stable. We will now show that (23) is more conservative than (10). Specifically, we will show that it is slightly more conservative than theorem 2 even if we assume that $\tilde{\Pi}_{11} \geq 0$ and $\tilde{\Pi}_{22} \leq 0$. Let

$$
\tilde{\Pi}=\left[\begin{array}{ccccc|ccccc}
I & 0 & 0 & 0 & 0 & 0 & 0 & 0 & 0 & 0 \\
0 & 0 & 0 & 0 & 0 & 0 & 0 & -I & 0 & 0 \\
0 & 0 & 0 & 0 & 0 & 0 & I & 0 & 0 & 0 \\
0 & 0 & 0 & I & 0 & 0 & 0 & 0 & 0 & 0 \\
0 & 0 & 0 & 0 & d^{2} I & 0 & 0 & 0 & 0 & 0 \\
\hline 0 & 0 & 0 & 0 & 0 & -I & 0 & 0 & 0 & 0 \\
0 & 0 & I & 0 & 0 & 0 & 0 & 0 & 0 & 0 \\
0 & -I & 0 & 0 & 0 & 0 & 0 & 0 & 0 & 0 \\
0 & 0 & 0 & 0 & 0 & 0 & 0 & 0 & -I & 0 \\
0 & 0 & 0 & 0 & 0 & 0 & 0 & 0 & 0 & -I
\end{array}\right]
$$

and

$$
T=\left[\begin{array}{c}
R \\
S \\
I
\end{array}\right], \quad U=\left[\begin{array}{cc}
U_{R} & 0 \\
0 & U_{S} \\
0 & 0
\end{array}\right], \quad V=\left[\begin{array}{c}
V_{R} \\
V_{S}
\end{array}\right] .
$$

This yields

$$
\begin{aligned}
& {\left[\begin{array}{cc}
T M & 0 \\
V M & 0 \\
\hline T & U \\
0 & I
\end{array}\right]^{*} \tilde{\Pi}\left[\begin{array}{cc}
T M & 0 \\
V M & 0 \\
\hline T & U \\
0 & I
\end{array}\right]=} \\
& {[]^{*}\left[\begin{array}{cccc}
R^{*} R+V_{R}^{*} V_{R}+d^{2} V_{S}^{*} V_{S} & S-S^{*} & 0 & U_{S} \\
S^{*}-S & -R^{*} R & -R^{*} U_{R} & 0 \\
0 & -U_{R}^{*} R & -\left(I+U_{R}^{*} U_{R}\right) & 0 \\
U_{S}^{*} & 0 & 0 & -I
\end{array}\right]\left[\begin{array}{ccc}
M & 0 & 0 \\
I & 0 & 0 \\
0 & I & 0 \\
0 & 0 & I
\end{array}\right]<0 .}
\end{aligned}
$$

Using Schur complements, we rewrite it as

$$
\left[\begin{array}{c}
M \\
I
\end{array}\right]^{*}\left[\begin{array}{cc}
R^{*} R+V_{R}^{*} V_{R}+d^{2} V_{S}^{*} V_{S}+U_{S} U_{S}^{*} & S-S^{*} \\
S^{*}-S & -R^{*}\left(I+U_{R} U_{R}^{*}\right)^{-1} R
\end{array}\right]\left[\begin{array}{c}
M \\
I
\end{array}\right]<0 .
$$


Note that (24) is very close to the IQC (23) proposed by Jönsson and Rantzer $[3,2]$ for slowly varying uncertainties, which is slightly more conservative, since

$$
I-U_{R} U_{R}^{*} \leq\left(I+U_{R} U_{R}^{*}\right)^{-1} .
$$

for all $U_{R}$. Note that main restriction in (23) is that it assumes semidefinite $\tilde{\Pi}_{22}$ and $\tilde{\Pi}_{11}$.

\section{Examples}

We will show the stability criterion on two examples. In both examples the improvements compared to previous IQC-based bounds are significant.

\subsection{Example I}

This example is from [3]. A simple SISO system is considered:

$$
\dot{x}(t)=(A+C \delta(t) B) x(t)=\left[\begin{array}{cc}
-0.21 & -1+0.8 \delta(t) \\
1 & 0
\end{array}\right] x(t)
$$

where it is assumed that $\delta(t) \in[-\gamma, \gamma]$. In the case the uncertainty parameter is constant, the system is asymptotically stable if and only if $\delta<1.25$, that is, when $\gamma<1.25$.

If $\delta$ is unbounded, then stability is assured if $|\delta|<0.261$. This bound is obtained as the inverse of the $\mathcal{H}_{\infty}$-norm of

$$
G(s)=D+C(s I-A)^{-1} B
$$

where

$$
\left[\begin{array}{ll}
A & B \\
C & D
\end{array}\right]=\left[\begin{array}{cc|c}
-0.21 & -1 & 0.8 \\
1 & 0 & 0 \\
\hline 0 & 1 & 0
\end{array}\right]
$$

We now apply theorem 2 using

$$
T(s)=\left[\begin{array}{c}
\frac{1}{s+1} \\
1
\end{array}\right] .
$$

This multiplier has the same pole as that in [3]. There the analysis using a frequency dependent IQC yields a stability bound of $|\dot{\delta}|<d=0.1$ for $\gamma=1$. Using (25) yields $d=0.1036$, which is in close agreement with [3].

If we instead choose

$$
T(s)=\left[\begin{array}{c}
\frac{1}{s+10} \\
1
\end{array}\right],
$$

we obtain $d=0.1456$ for $\gamma=1$, assuming $\tilde{\Pi}_{11} \geq 0$ and $\tilde{\Pi} \leq 0$. Relaxing the requirements on $\tilde{\Pi}$ and instead assuming $\Pi_{11}(j \omega) \geq 0$ and $\Pi_{22}(j \omega) \leq 0$, we obtain $d=0.3111$ for $\gamma=1$ with (26). Figure 2 shows the stability bounds, $d$, as a function of $\gamma$. Instability has been verified for $\gamma=0.41$ without any bounds 


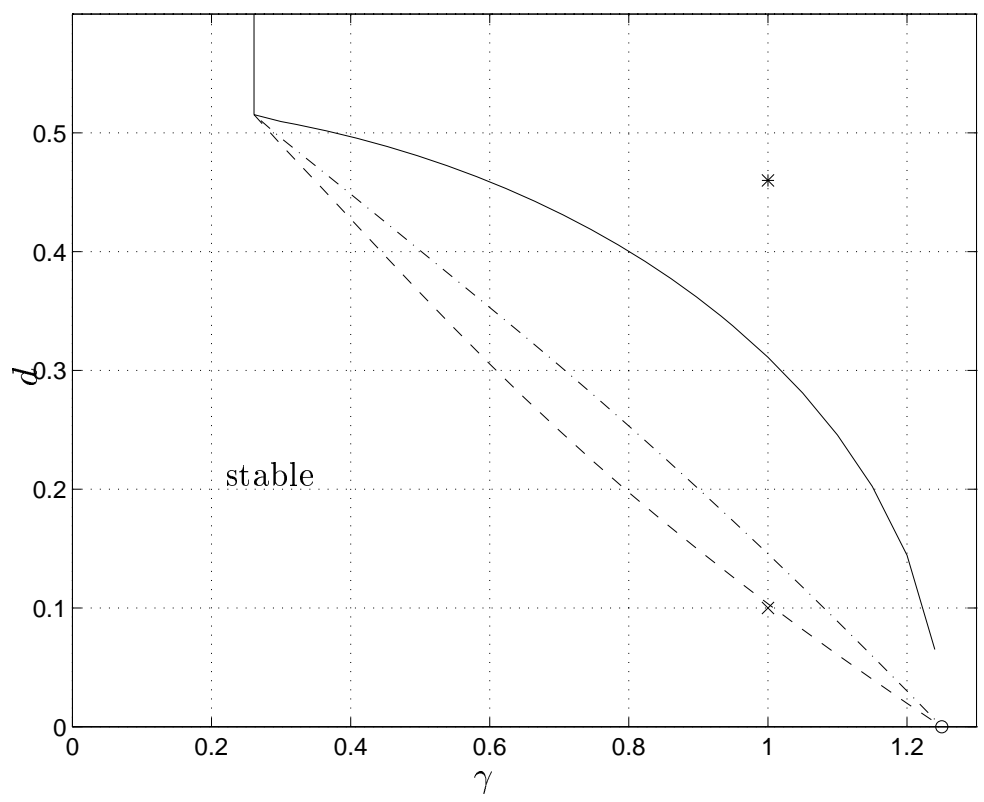

Figure 2: Example I: Stability bounds $d$ as a function of $\gamma$. The system is stable of $\delta(t) \in[-\gamma, \gamma]$ and $|\dot{\delta}(t)| \leq d$. The lower, dashed line uses $T$ as defined in (25) while the middle, dashed-dotted line uses (26). Both assume semidefinite $\tilde{\Pi}_{i i}$. The upper, solid line uses the relaxed constraints on $\tilde{\Pi}_{i i}$ and the multiplier defined by (26). The cross $(\times)$ marks the bound given in [3]. The circle (o) at $\gamma=1.25$, denotes the bound obtained with constant $\delta$. Arbitrarily fast variations in $\delta$ can be allowed if $|\delta|<0.261$, marked by the vertical line. The criteria show stability in the area below and to the left of the lines. The asterisk $(*)$ at $\gamma=1$ and $d=0.46$, shows a case for which instability has been verified. 
on $\dot{\delta}$ and $d=0.46$ for $\gamma=1$; these bounds are (probably) not the true bounds though.

The lower bound could probably be improved by using a basis multiplier, $T$, of high order. However, this leads to an increased computational load, as well as that the condition number of the required $\tilde{\Pi}$ usually increases. This may cause numerical problems, which could result in worse bounds even if this is not theoretically true.

\subsection{Example II}

This example is has been analyzed in [4] using IQCs. We consider the ship steering dynamics as in Example 9.6 in [1]. The dynamics can be approximated by the Nomoto model

$$
\begin{aligned}
\dot{x}(t) & =v(t)(-a x(t)+b v(t) \nu(t)) \\
\dot{\psi}(t) & =x(t)
\end{aligned}
$$

where $\psi$ denotes the heading of the ship, $\nu$ denotes the rudder angle and $v$ is the speed of the ship. It is assumed that $v(t) \geq 0$. We will, as in [1], study the stability of the ship dynamics for an unstable tanker with $a=-0.3$ and $b=0.8$, which is controlled by a $\mathrm{PD}$ regulator

$$
v=-K \psi \quad \text { where } \quad K(s)=k\left(1+T_{d} s\right)
$$

with $k=2.5$ and $T_{d}=0.86$.

The closed loop system can be described by

$$
x(t)=\left(v(t) I_{2}\right) G_{0}(s) x(t)
$$

with

$$
G_{0}(s)=\left[\begin{array}{cc}
-a / s & b \\
-K(s) / s^{2} & 0
\end{array}\right] .
$$

This system is not strictly stable and instead we replace it for the purpose of analysis by

$$
G(s)=\left(I+G_{0}(s)\right)\left(I-G_{0}(s)\right)^{-1}=D+C(s I-A)^{-1} B
$$

where

$$
\left[\begin{array}{ll}
A & B \\
C & D
\end{array}\right]=\left[\begin{array}{cc|cc}
-\left(a+b k T_{d}\right) & -b k & -2 & -2 b \\
1 & 0 & 0 & 0 \\
\hline a+b k T d & b k & 1 & 2 b \\
k T_{d} & k & 0 & 1
\end{array}\right]
$$

and

$$
\delta(t)=\frac{v(t)-v_{\text {nom }}}{v(t)+v_{\text {nom }}} \in[-1,1],
$$

where $v_{\text {nom }}$ is the nominal speed of the ship. For the transformed system, $d$ is solved as a function of $\gamma$ such that the system is verified to be stable when

$$
|\delta| \leq \gamma \quad \text { and } \quad|\dot{\delta}| \leq d .
$$




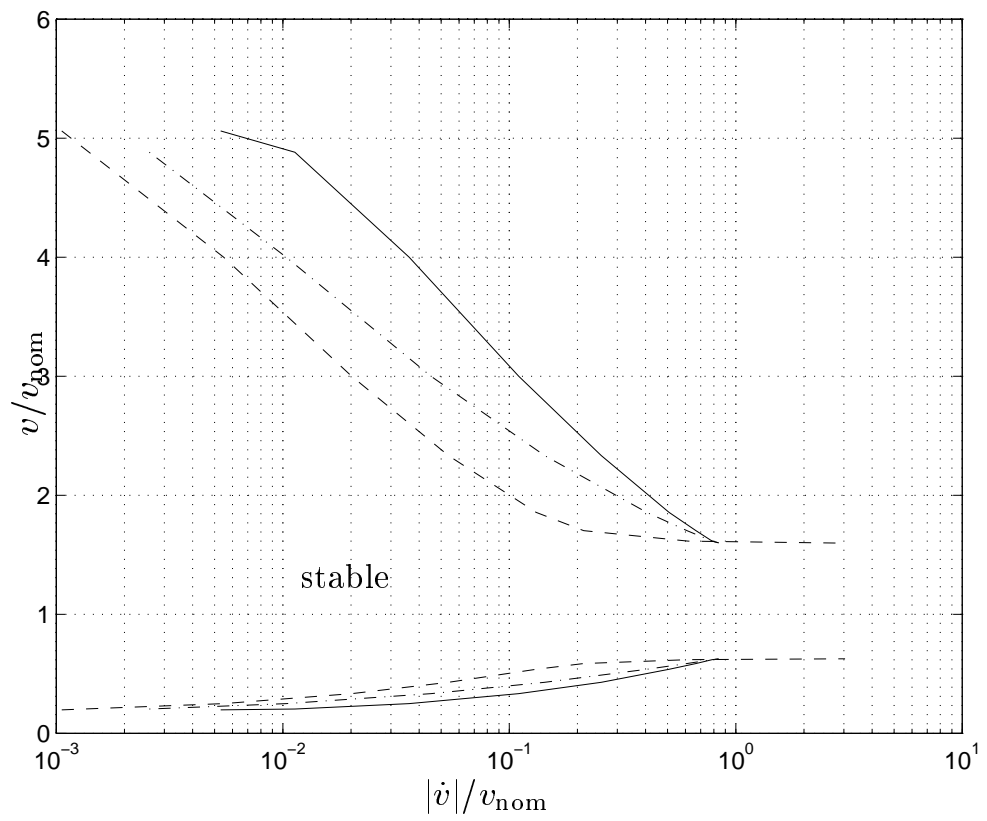

Figure 3: Example II: Stability bounds for $v$ and $\dot{v}$ using different IQC criteria. The solid lines show the proven stability bounds using $T$ as defined in (27) assuming $\Pi_{11}(j \omega) \geq 0$ and $\Pi_{22}(j \omega) \leq 0$ for all $\omega \in \mathbb{R}$, while the dash-dotted lines assume $\tilde{\Pi}_{11} \geq 0$ and $\tilde{\Pi}_{22} \leq 0$. The dashed lines mark the bounds given in [4].

Then

$$
v=\frac{1+\delta}{1-\delta} v_{\text {nom }} \quad \text { and } \quad \dot{v}=\frac{2 v_{\text {nom }}}{(1-\delta)^{2}} \dot{\delta},
$$

and the system is assured to be stable when

$$
\begin{aligned}
& \frac{1-\gamma}{1+\gamma} \leq \frac{v(t)}{v_{\text {nom }}} \leq \frac{1+\gamma}{1-\gamma} \\
& \frac{|\dot{v}|}{v_{\text {nom }}} \leq \frac{2 d}{(1+\gamma)^{2}}
\end{aligned}
$$

We analyze the system using

$$
T=\left[\begin{array}{c}
I_{2} \\
(s+1)^{-1} I_{2}
\end{array}\right] .
$$

Assuming that $\delta$ is constant, stability can be assured for $|\delta| \leq 0.7028$ or, equivalently, $0.1745 \leq v \leq 5.7296$. This is close to the true stability bound, $v / v_{\text {nom }} \geq-a /\left(b k T_{d}\right)=0.1744$.

Figure 3 shows the area in the $(v, \dot{v})$-plane where stability is proven using the different criteria. 


\section{Conclusions}

A new integral quadratic constraint (IQC) criterion for verifying stability of systems with slowly varying parameters has been derived. The new criterion shows significant improvements in bounds than previous criteria. This is illustrated by two examples. We have shown that the new criterion is always less conservative than the old one if the same basis multipliers are used.

\section{References}

[1] K. J. Åström and B. Wittenmark. Adaptive Control. Addison Wesley Series in Electrical and Computer Engineering: Control Engineering. Addison Wesley, 1989.

[2] U. Jönsson. Robustness Analysis of Uncertain and Nonlinear Systems. PhD thesis, Lund Institute of Technology, Lund, Sweden, 1996.

[3] U. Jönsson and A. Rantzer. System with uncertain parameters - timevariations with bounded derivatives. In Proceedings of the 33rd Conference on Decision and Control, volume 3, pages 3074-3079, Lake Buena Vista, Florida, December 1994.

[4] U. Jönsson and A. Rantzer. Systems with uncertain parameters - time variations with bounded derivatives. International Journal of Robust and Nonlinear Control, 1997. submitted and accepted.

[5] A. Megretski and A. Rantzer. System analysis via integral quadratic constraints. IEEE Transactions on Automatic Control, 42(6):819-830, June 1997.

[6] A. Rantzer. A note on the Kalman-Yacubovich-Popov lemma. In Proceedings of the 3rd European Control Conference, volume 3, part 1, pages 1792-1795, Rome, Italy, September 1995.

[7] J. C. Willems. The Analysis of Feedback Systems. MIT Press, Cambridge, MA, 1971.

[8] V. A. Yakubovich. A frequency theorem for the case in which the state and control spaces are Hilbert spaces with an application to some problems of optimal controls - Part I-II. Sibirskii Mat. Zh., 15(3):639-668, 1974. English translation in Siberian Math. J. 